\title{
Contemporary evaluation of the causes of cardiac tamponade: Acute and long-term outcomes
}

\author{
Ady Orbach, Jorge E. Schliamser, Moshe Y. Flugelman, Barak Zafrir \\ Department of Cardiovascular Medicine, Lady Davis Carmel Medical Center and the Ruth \\ and Bruce Rappaport School of Medicine, Technion-Israel Institute of Technology, Haifa, Israel
}

\begin{abstract}
Background: Cardiac tamponade is a life-threatening state that complicates various medical conditions. The contemporary interventional era may have led to changes in clinical characteristics, causes and outcomes of cardiac tamponade.

Methods: We investigated all patients diagnosed with cardiac tamponade, based on clinical and echocardiographic findings, at a single medical center between the years 2000 and 2013. Data on medical history, index hospitalizations, pericardial fluid etiologies, and acute and long-term outcomes were collected.
\end{abstract}

Results: Cardiac tamponade was observed in 83 patients (52\% females). Major etiologies included complications of percutaneous cardiac interventions (36\%) and malignancies (primarily lung cancer; 23\%), infectious/inflammatory causes (15\%) and mechanical complications of myocardial infarction (12\%). Sixteen (19\%) patients died during the index hospitalization. Acute presentation of symptoms and lower quantity of effusion were associated with in-hospital mortality ( $p=0.045$ and $p=0.007)$. Tamponade secondary to malignancy was associated with the most substantial increment in post-discharge mortality (from 16\% in-hospital to 68\% 1-year mortality). During the mean follow-up of 45 months, 39 (45\%) patients died. Malignancies, mechanical complications of myocardial infarction and bleeding/coagulation abnormalities were etiologies associated with poor survival (80\% mortality during follow-up). Tamponade secondary to complications of percutaneous cardiac interventions or infectious/inflammatory causes were associated with significantly lower mortality (28\% and 17\%; log rank $p<0.001$ ).

Conclusions: In a contemporary cohort, complications of percutaneous cardiac intervention replaced malignant diseases as the leading cause of cardiac tamponade. Nevertheless, these iatrogenic complications were associated with a relatively favorable outcome compared to tamponade induced by complications of myocardial infarction, coagulation abnormalities and malignant diseases. (Cardiol J 2016; 23, 1: 57-63)

Key words: cardiac tamponade, procedural complications, outcome

\section{Introduction}

Cardiac tamponade is the accumulation of fluid in the pericardial space, leading to rapid fall in cardiac output, hypotension and systemic hypoperfu- sion [1]. Cardiac tamponade is a life-threatening condition that requires immediate intervention [1]. The clinical presentation is influenced by the rate at which pericardial fluid accumulates, deriving from the specific etiology of the tamponade [2].

Address for correspondence: Barak Zafrir, MD, Cardiovascular Department, Lady Davis Carmel Medical Center, 7 Michal St., Haifa, Israel, tel: +972-48250285, fax: +972-99560390, e-mail: barakzmd@gmail.com 
Cardiac tamponade occurs in diverse clinical situations, including complications of both acute and chronic medical conditions, as well as iatrogenic complications of surgical and percutaneous cardiac interventional procedures [3]. Presentation may be rapid: after chest wall trauma, after aortic or cardiac rupture, or due to complications of cardiac procedures, necessitating prompt diagnosis and treatment. However, complications of medical conditions such as infections, metastatic malignancies, connective tissue diseases, uremia, hypothyroidism or drug side effects, can be associated with pericarditis or pericardial effusion, which may progress more gradually to overt tamponade [3].

In recent years, applications of interventional cardiovascular diagnostic and therapeutic procedures have increased, with the introduction of new and complex technologies for structural heart disease and electrophysiology $[4,5]$. The use of antiplatelets and anticoagulation in clinical medicine is also expanding. On the other hand, traditional etiologies of pericarditis, such as tuberculosis, are decreasing in western societies. These developments may have conceivably changed the characteristics and implications of cardiac tamponade in clinical medicine. Accordingly, the aim of the current study was to evaluate the clinical presentation, causes, and short- and long-term outcomes of cardiac tamponade, in a cohort of medical patients representing the contemporary interventional era.

\section{Methods}

\section{Study cohort}

The study population included all consecutive patients diagnosed with a first episode of cardiac tamponade during hospitalization at a single university affiliated medical center (Carmel Medical Center, Haifa, Israel), between the years 2000 and 2013. Tamponade cases secondary to cardiac surgery or chest-wall trauma were excluded from the cohort.

Baseline characteristics including demographic and clinical data, comorbidities, blood tests results and echocardiographic parameters were documented for all patients. Cardiac tamponade was diagnosed based on customary echocardiographic evidence of tamponade physiology, such as right ventricular diastolic collapse, in addition to documented clinical evidence of tamponade or hemodynamic failure. All patients underwent pericardiocentesis or urgent surgery. Patients' electronic files were reviewed retrospectively for documentation of the clinical presentation of the cardiac tamponade, usage of anticoagulants and the probable underlying etiologies.

The incidence of cardiac tamponade complicating percutaneous coronary and valvular interventions was calculated by analyzing the computerized database of the cardiac catheterization unit. In addition, we have estimated the total number of electrophysiologic interventional procedures performed during the study period, as no computerized data were available regarding the volume of these procedures during the initial years of the study.

Symptomatic presentation was classified as acute (symptoms at the day of tamponade presentation), subacute (1-7 days) or chronic (more than a week before tamponade presentation). Pericardial effusion quantity, as estimated by echocardiography during tamponade diagnosis, and quantification of the pericardial fluid drained, were noted.

Mortality during index hospitalization, as well as 30-day and 1-year mortality, were ascertained through July 2014 for all subjects, by reviewing computerized records of the health maintenance organization. The study protocol was approved by the Lady Davis Carmel Medical Center (Haifa, Israel) Institutional Review Board.

\section{Statistical analysis}

Continuous data are presented as mean \pm standard deviation (SD) and categorical variables as numbers and percentages. The independentsamples T-test was used to compare continuous variables, and the $\chi^{2}$ test was used to compare categorical variables. Fisher's exact test was used in cases of small sample sizes. Survival curves were plotted for each of the main tamponade etiologies by the Kaplan-Meier method using the log-rank test for comparison between variables.

The results were considered statistically significant when the 2 -sided $\mathrm{p}$-value was $<0.05$. SPSS statistical software version 20.0 was used to perform all statistical analyses.

\section{Results}

Eighty-three consecutive patients diagnosed with a first episode of cardiac tamponade during the years 2000 to 2013 were included in the analysis after exclusion of tamponade cases secondary to cardiac surgery or chest-wall trauma. Mean age of the study population was $67 \pm 17$ years, and $52 \%$ were females. Baseline characteristics are presented in Table 1. Preserved left ventricular ejection fraction was noted in $52(63 \%)$ patients. Acute presentation of symptoms was present in 
Table 1. Baseline characteristics of the study patients.

\begin{tabular}{|c|c|}
\hline Variable & $\begin{array}{l}\text { Number (\%) } \\
\text { or mean } \pm \text { SD }\end{array}$ \\
\hline Age & $67.1 \pm 16.8$ \\
\hline Gender (female) & $43(52 \%)$ \\
\hline Smoking & $23(28 \%)$ \\
\hline Diabetes mellitus & $30(36 \%)$ \\
\hline Hypertension & $54(65 \%)$ \\
\hline Coronary artery disease & $30(36 \%)$ \\
\hline $\begin{array}{l}\text { Chronic obstructive } \\
\text { pulmonary disease }\end{array}$ & $7(8 \%)$ \\
\hline Chronic renal failure & $16(19 \%)$ \\
\hline Known malignancy & $16(19 \%)$ \\
\hline Creatinine [mg/dL] & $1.40 \pm 1.07$ \\
\hline Urea $[\mathrm{mg} / \mathrm{dL}]$ & $59 \pm 37$ \\
\hline Hemoglobin $[\mathrm{g} / \mathrm{dL}]$ & $12.0 \pm 1.8$ \\
\hline \multicolumn{2}{|c|}{ Symptomatic presentation: } \\
\hline Acute & $44(53 \%)$ \\
\hline Subacute & $34(41 \%)$ \\
\hline Chronic & $5(6 \%)$ \\
\hline \multicolumn{2}{|c|}{$\begin{array}{l}\text { Pericardial effusion quantity } \\
\text { (estimation by echo): }\end{array}$} \\
\hline Mild & $5(6 \%)$ \\
\hline Mild-moderate & $6(7 \%)$ \\
\hline Moderate & $12(14 \%)$ \\
\hline Moderate-large & $4(5 \%)$ \\
\hline Large & $45(57 \%)$ \\
\hline Unknown & $9(11 \%)$ \\
\hline
\end{tabular}

$44(53 \%)$. The majority of patients $(96 \%)$ were treated by pericardiocentesis. The volume of effusion drained ranged from $15 \mathrm{~mL}$ to $3 \mathrm{~L}$ (median $600 \mathrm{~mL}$ ), and $13(16 \%)$ patients required blood product transfusions.

Various etiologies contributing to tamponade episodes are presented in Table 2. Complications of cardiovascular percutaneous interventional procedures were the most common causes of tamponade, observed in $29(36 \%)$ patients, including complications of percutaneous coronary and valvular interventions as well as electrophysiological procedures.

A total of 22,910 percutaneous coronary and valvular procedures were performed between January 1, 2000, and December 31, 2013. Among them there were 11,699 therapeutic interventions, of which 21 were complicated by cardiac tamponade $(0.18 \%)$. In addition, the estimated number of electrophysiological procedures during this period
Table 2. Etiologies of cardiac tamponade.

\begin{tabular}{lc}
\hline Tamponade etiology & Number (\%) \\
\hline Cardiac percutaneous interventional complications: \\
PCl complications & $12(14.5 \%)$ \\
TAVR/BAV complications & $3(4 \%)$ \\
Pacemaker insertion/ablation & $14(17 \%)$ \\
complications: & \\
$\quad$ Permanent pacemaker/ICD & 6 \\
implantation & \\
Temporary pacemaker & 6 \\
electrode extraction & 2 \\
Radiofrequency ablation & $19(23 \%)$ \\
Malignancy: & 11 \\
Lung & 2 \\
Breast & 1 \\
Lymphoma & 5 \\
Other/unknown origin & $12(14.5 \%)$ \\
Infectious/inflammatory: & 2 \\
Bacterial infection & $10(12 \%)$ \\
Acute myocardial infarction & $8(10 \%)$ \\
complications & $5(6 \%)$ \\
Unknown/idiopathic & \\
Bleeding/coagulation & \\
abnormalities & \\
\hline BAV & \\
\hline
\end{tabular}

BAV - balloon aortic valvuloplasty; ICD — implantable cardioverter defibrillator; $\mathrm{PCl}$ - percutaneous coronary intervention; TAVR trans-aortic valve replacement

was 3,920 , of which 8 cases were complicated by cardiac tamponade $(0.20 \%)$. We considered cardiac tamponade secondary to temporary wire extraction a coronary/valvular procedural complication.

Malignancies, primarily lung cancer, were the second leading etiology of cardiac tamponade (19 [23\%] patients). Of the infectious/inflammatory causes (12 [14.5\%] patients), only 2 cases were secondary to a diagnosed bacterial infection. Acute myocardial infarction (MI) complications were present in $10(12 \%)$ cases. Bleeding and coagulation disorders were additional notable causes. Five patients suffering from tamponade who were concomitantly treated with anticoagulation (warfarin), presented with severely abnormal coagulation tests (international normalized ratio levels 6.3-23), suggesting bleeding as the direct cause of tamponade. In addition, of the 6 patients with tamponade occurring immediately after an extraction of a temporary intravenous pacemaker wire, 3 were treated with glycoprotein (GP) IIb/IIIa inhibitor (eptifibatide) adjacent to the event.

Acute presentation of symptoms was noted in all cases of tamponade secondary to MI complica- 
Table 3. Association of baseline characteristics with in-hospital mortality.

\begin{tabular}{lccc}
\hline Variable & Alive $(\mathbf{n}=67)$ & In-hospital mortality (n = 16) & P \\
\hline Age & $67 \pm 18$ & $69 \pm 13$ & 0.548 \\
Gender (female) & $36(54 \%)$ & $7(44 \%)$ & 0.581 \\
Smoking & $20(30 \%)$ & $3(19 \%)$ & 0.537 \\
Diabetes mellitus & $26(39 \%)$ & $4(25 \%)$ & 0.392 \\
Hypertension & $44(66 \%)$ & $10(63 \%)$ & 0.812 \\
Coronary artery disease & $24(36 \%)$ & $6(38 \%)$ & 0.900 \\
Chronic renal failure & $11(16 \%)$ & $5(31 \%)$ & 0.287 \\
Known malignancy & $14(21 \%)$ & $2(13 \%)$ & 0.725 \\
Moderate/severe reduced LVEF & $6(9 \%)$ & $2(25 \%)$ & 0.215 \\
Blood product transfusion & $12(18 \%)$ & $1(7 \%)$ & 0.267 \\
Acute presentation of symptoms & $32(48 \%)$ & $12(75 \%)$ & 0.045 \\
Creatinine [mg/dL] & $1.39 \pm 1.16$ & $1.44 \pm 0.48$ & 0.783 \\
Urea [mg/dL] & $57 \pm 36$ & $68 \pm 42$ & 0.375 \\
Hemoglobin [g/dL] & $11.9 \pm 1.9$ & $12.5 \pm 1.6$ & 0.184 \\
Effusion quantity [mL] & $815 \pm 602$ & $356 \pm 333$ & 0.007 \\
\hline
\end{tabular}

LVEF - left ventricular ejection fraction

tions and in $93 \%$ of complications of percutaneous cardiac interventions. In addition, acute or subacute presentation was reported in all cases of tamponade secondary to bleeding/coagulation abnormalities. In contrast, $81 \%$ of patients with malignancy associated with cardiac tamponade had subacute or chronic presentation of symptoms.

During the index hospitalization, the all-cause mortality rate was $19 \%$ (16 patients). In-hospital mortality was associated with acute presentation of symptoms and smaller quantity of pericardial effusion drained, but was not associated with common comorbidities (Table 3). The 30-day mortality rate was $24(29 \%)$ patients. During a mean follow-up period of $45 \pm 50$ months (median 30 months), a total of $39(47 \%)$ patients died.

Short- and long-term mortality rates, according to the various causes of cardiac tamponade, are presented in Figure 1. Complications of acute MI and bleeding or coagulation disorders were the causes associated with the worst survival $(80 \%$ 1-year mortality). Tamponade secondary to malignancies was associated with the most substantial increase in post-discharge mortality (from 16\% in-hospital to $68 \%$ 1-year mortality). Cardiac tamponade cases without any known identified etiology were evident in 8 patients, and were not associated with mortality events during follow-up. Cumulative survival rates are presented in Figure 2, differentiating between etiologies associated with very poor as opposed to better long-term survival.
The log-rank test p-value for the overall comparison between tamponade etiologies was $<0.001$. In addition, patients with tamponade secondary to complications of percutaneous cardiac interventions had significantly better long-term survival than each of the 3 etiologies associated with poor survival (bleeding/coagulation abnormalities, malignancies, and MI complications); log-rank $\mathrm{p}<0.01$ for each pairwise comparison.

\section{Discussion}

The present study, which comprised a contemporary cohort, revealed iatrogenic complications of percutaneous cardiac procedures to be the most prevalent etiology for cardiac tamponade. This finding contrasts with previous clinical studies, which reported malignant diseases as the most common cause of pericardial tamponade, reaching 30-60\% of cases [6-11]. This shift in tamponade etiology is apparently due to the increase in recent years in the overall number and in the complexity of percutaneous intra-cardiac procedures, as well as the introduction of interventional techniques such as trans-catheter valve replacement and catheter ablation for atrial fibrillation, all reported to be possibly complicated by cardiac tamponade [12-15].

In the current study, $0.18 \%$ of percutaneous coronary and valvular interventions were complicated by cardiac tamponade. This is in accordance with recent data reporting an incidence of tampon- 


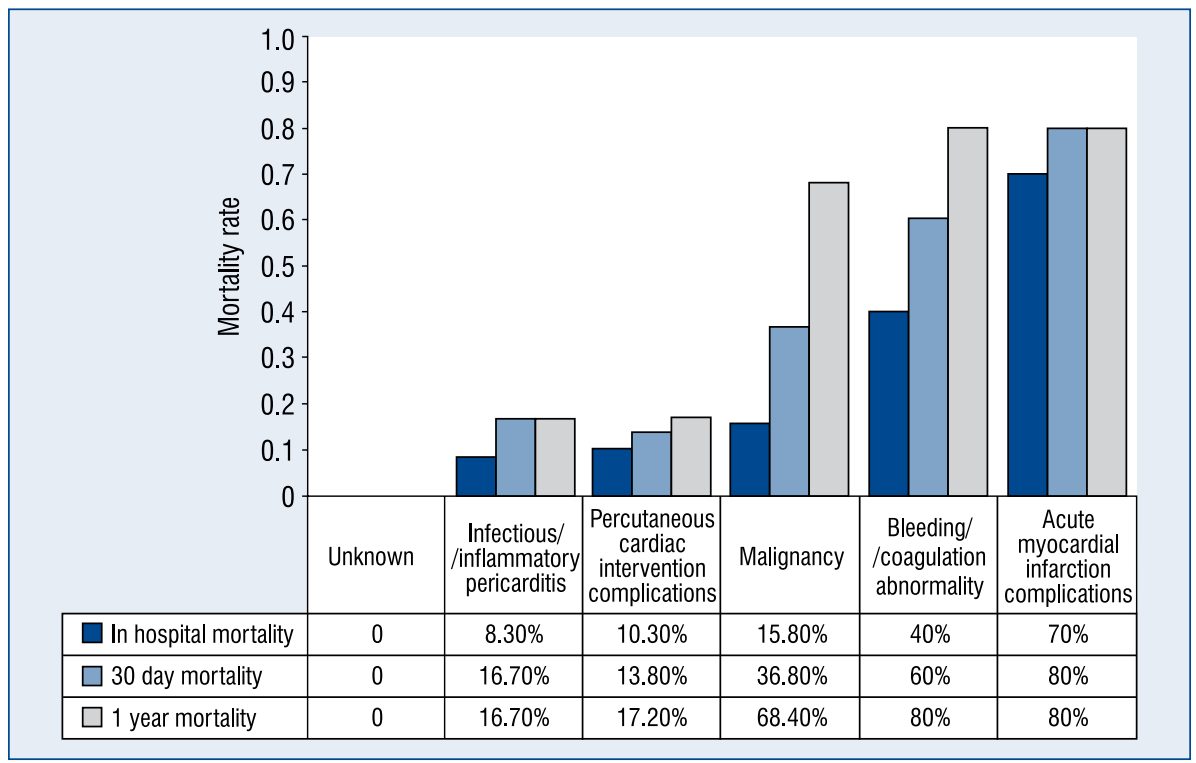

Figure 1. Mortality rates according to different causes of cardiac tamponade.

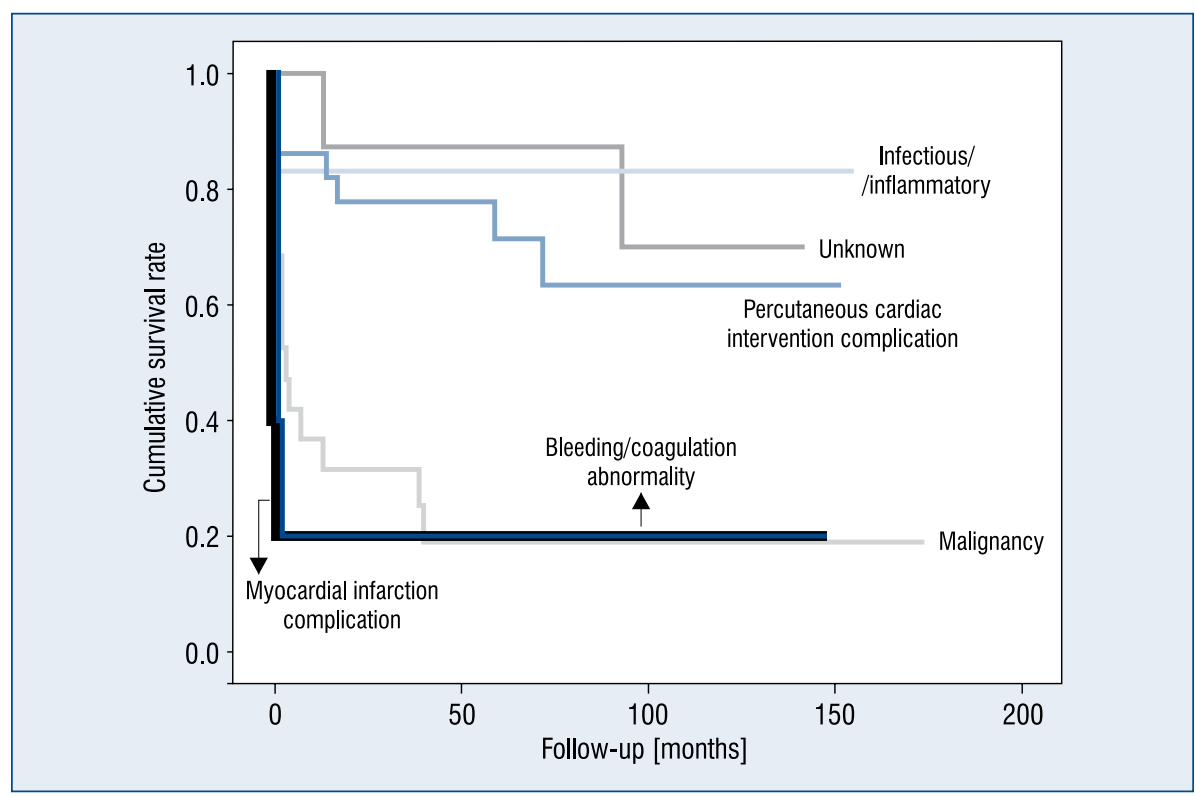

Figure 2. Long-term survival, according to cardiac tamponade etiologies.

ade complicating percutaneous coronary interventions ranging $0.036-0.38 \%$ in several previously published series [13]. The incidence of cardiac tamponade complicating electrophysiological procedures is less comparable due to the variability in type and complexity of procedures performed at different centers. Some technically demanding procedures such as atrial fibrillation ablation are associated with higher rates of tamponade, and were not performed in our center [12].
Despite its being the main etiology, iatrogenic cardiac tamponade secondary to percutaneous cardiac procedures showed relatively low in-hospital and post hospitalization mortality, probably due to prompt diagnosis and treatment of these conditions. On the other hand, the highest mortality rates, both during hospitalization and after, were among tamponade cases secondary to mechanical complications of acute MI. An example is free wall rupture, a fatal complication that has decreased in 
frequency in the percutaneous coronary revascularization era.

The origins of malignant pericardial effusions include solid tumors and hematological malignancies. Similar to our population, lung cancer was the most common origin of the malignant processes reported in studies summarizing clinical outcomes of malignant effusions [6, 8]. The mortality pattern of patients with malignancy-induced tamponade showed a low in-hospital rate, with progressive increment during the year after the event. This may be attributable to the more gradual accumulation of the malignant effusion, which reduces the risk for a life threatening presentation, and enables treating the tamponade while the basic malignant disease advances. Similar outcomes have been shown in other studies that reported very low 1-year survival rates, ranging from $10 \%$ to $27 \%$ in malignant pericardial effusion $[6,16]$. Accordingly, malignancy induced tamponade may indicate an ominous sign of advanced cancer.

The clinical indications for antiplatelet and anticoagulant therapy have expanded in recent years. Poor anticoagulation control, as seen in several patients in the current study, may precipitate the development of hemopericardium, and is well documented in the literature as a possible cause of tamponade $[17,18]$. In addition, an increasing number of patients are treated nowadays by novel oral anticoagulants (NOACs), for which assays to monitor the coagulation status are not yet commonly available. This may expose patients to lifethreatening bleeding, including pericardial effusion, which was recently reported in patients receiving NOACs $[19,20]$.

The use of adjuvant therapy with platelet GP IIb/IIIa inhibitors in patients with acute coronary syndromes has increased markedly prior to complex coronary interventions, and is another possible contributor to tamponade in cases of coronary perforation. In the current study, we report on 3 cases in which cardiac tamponade occurred following extraction of intravenous temporary pacing wire in patients who received GP IIb/IIIa inhibitors after invasive cardiac procedures. In these cases, the removal of the temporary pacing wire probably exposed the right ventricular perforation site, which led to the cardiac tamponade. Such cases, although rarely reported in the literature, might not be uncommon, and highlight the importance of careful management of patients with temporary pacing wires, including avoidance of frequent lead repositioning, usage of balloon-tipped pacing wires, monitoring arterial blood pressure for hemodynamic collapse, and accounting for the elimination half time of the GP IIb/IIIa inhibitor antiplatelet agent before removal of the temporary pacing wire $[21,22]$.

\section{Limitations of the study}

Potential limitations of the current study include the relatively low number of tamponade cases and the retrospective design. Moreover, the study population represents a single medical center, limiting the generalization of the results. In addition, except for fluid quantity, we did not analyze the characteristics of the pericardial effusion and the diagnostic evaluation process, due to incomplete documentation of data in patients' medical records.

\section{Conclusions}

In conclusion, pericardial tamponade derives from a wide range of etiologies with variable clinical outcomes. In a contemporary cohort of medical patients, complications of percutaneous cardiac interventions replaced malignant diseases as the leading cause of cardiac tamponade. Nevertheless, these iatrogenic procedural complications were associated with relatively favorable in-hospital and 1-year outcome. Traditional causes of tamponade, such as complications of acute MI and malignant diseases, continue to be associated with poor outcome, as does tamponade associated with the usage of antiplatelet and anticoagulant therapies. The frequency of the latter may rise consequently to the expanding clinical indications and the growing number and complexity of percutaneous cardiac interventions.

\section{Conflict of interests: None declared}

\section{References}

1. Spodick DH. Current concepts: Acute cardiac tamponade. N Engl J Med, 2003; 349: 684-690.

2. Grecu L. Cardiac tamponade. Int Anesthesiol Clin, 2012; 50: 59-77.

3. Bodson L, Bouferrache KL, Vieillard-Baron A. Cardiac tamponade. Curr Opin Crit Care, 2011; 17: 416-424.

4. Langabeer JR, Henry TD, Kereiakes DJ et al. Growth in percutaneous coronary intervention capacity relative to population and disease prevalence. J Am Heart Assoc, 2013; 2: e000370.

5. Bradshaw PJ, Stobie P, Knuiman MW, Briffa TG, Hobbs MS. Trends in the incidence and prevalence of cardiac pacemaker insertions in an ageing population. Open Heart, 2014; 1: e000177.

6. Burazor I, Imazio M, Markel G, Adler Y. Malignant pericardial effusion. Cardiology, 2013; 124: 224-232.

7. De Ceuninck M, Demedts I, Trenson S. Malignant cardiac tamponade. Acta Cardiol, 2013; 68: 505-507.

8. Refaat M, Katz W. Neoplastic pericardial effusion. Clin Cardiol, 2011; 34: 593-598. 
9. Cornily JC, Pennec PY, Castellant P et al. Cardiac tamponade in medical patients: A 10-year follow-up survey. Cardiology, 2008; 111: 197-201.

10. Gibbs CR, Watson RDS, Singh SP, Lip GYH. Management of pericardial effusion by drainage: A survey of 10 years' experience in a city centre general hospital serving a multiracial population. Postgrad Med J, 2000; 76: 809-813.

11. Colombo A, Olson HG, Egan J, Gardin JM. Etiology and prognostic implications of a large pericardial effusion in men. Clin Cardiol, 1988; 11: 389-394.

12. Holmes DR, Nishimura R, Fountain R, Turi ZG. Iatrogenic pericardial effusion and tamponade in the percutaneous intracardiac intervention era. J Am Coll Cardiol Cardiovasc Interv, 2009; 2: 705-717.

13. Stathopoulos I, Kossidas K, Panagopoulos G, Garratt K. Cardiac tamponade complicating coronary perforation during angioplasty: Short-term outcomes and long-term survival. J Invasive Cardiol, 2013; 25: 486-491.

14. Georgiadou P, Karavolias G, Sbarouni E, Adamopoulos S, Malakos J, Voudris V. Coronary artery perforation in patients undergoing percutaneous coronary intervention: A single-centre report. Acute Cardiac Care, 2009; 11: 216-221.

15. Patel VG, Brayton KM, Tamayo A et al. Angiographic success and procedural complications in patients undergoing percutaneous coronary chronic total occlusion interventions: A weighted meta- analysis of 18,061 patients from 65 studies. J Am Coll Cardiol Cardiovasc Interv, 2013; 6: 128-136.

16. Halfdanarson TR, Hogan WJ, Moynihan TJ. Oncologic emergencies: Diagnosis and treatment. Mayo Clin Proc, 2006; 81: 835-848.

17. Fel SC, Rubin IL, Enselberg CD, Hurwitt ES. Anticoagulantinduced hemopericardium with tamponade and its occurrence in the absence of myocardial infarction or pericarditis. $\mathrm{N}$ Engl J Med, 1965; 272: 670e674.

18. Hong YC, Chen YG, Hsiao CT, Kuan JT, Chiu TF, Chen JC. Cardiac tamponade secondary to haemopericardium in a patient on warfarin. Emerg Med J, 2007; 24: 679-680.

19. Abdallah $\mathrm{M}$, Abdallah $\mathrm{T}$, Abi Rafeh $\mathrm{N}$ et al. A sanguineous pleuro pericardial effusion in a patient recently treated with dabigatran. Heart Lung, 2015; 44: 209-211.

20. Barton CA, McMillian WD, Raza SS, Keller RE. Hemopericardium in a patient treated with dabigatran etexilate. Pharmacotherapy, 2012; 32: e103-e107.

21. Jeilan M, Richardson G, Gershlick A. Transvenous pacing causing tamponade in patients receiving glycoprotein IIb/IIIa inhibitors for percutaneous coronary intervention. J Invasive Cardiol, 2007; 19: E40-E42.

22. Mahon L, Bena JF, Morrison SM, Albert NM. Cardiac tamponade after removal of temporary pacer wires. Am J Crit Care, 2012; 21: $432-440$. 